\title{
Limitaciones de la psicomotricidad en la etapa preescolar durante el COVID-19
}

\author{
Limitations of psychomotor skills in preschool during COVID-19
}

Limitações das habilidades psicomotoras na pré-escola durante o COVID-19

\section{Delia Rocío Chero-Pacheco}

dchero@ucvvirtual.edu.pe

https://orcid.org/0000-0002-0168-0744

Universidad César Vallejo, Lima-Perú

Rossana Delia Mezarina Castañeda

rmezarinac@ucvvirtual.edu.pe

https://orcid.org/0000-0003-2919-4809

Universidad César Vallejo, Lima-Perú

\begin{abstract}
Alfredo Magallanes-Taype
amagallanest13@ucvvirtual.edu.pe https://orcid.org/0000-0001-9094-7342

Universidad César Vallejo, Lima-Perú
\end{abstract}

Melquiades Carmelino Silvestre Bohorquez

msilvestre05051@ucvvirtual.edu.pe https://orcid.org/0000-0002-0568-6369

Universidad César Vallejo, Lima-Perú

Artículo recibido 18 de noviembre 2021, arbitrado y aceptado 6 de diciembre 2021 y publicado 3 de marzo 2022

\section{RESUMEN}

El cierre de las escuelas por la pandemia del COVID-19, afectó severamente a los niños en edad preescolar, debido a que ellos fueron los más vulnerables porque dependían de un adulto para orientarlos en sus actividades académicas, por tal razón, su desarrollo normal escolar resultó interrumpido. El objetivo de este estudio es explicar las limitaciones de la psicomotricidad en la etapa preescolar durante el COVID-19. Empleándose el método descriptivo de revisión bibliográfica, la consulta de la literatura académica brindó como resultados coincidencias en dos temas: las dificultades psicológicas y de los padres para ayudarlos en el desarrollo de sus actividades psicomotrices. Se concluyó que existieron limitaciones para desarrollar la psicomotricidad; sin embargo, los docentes se adaptaron a la nueva normalidad y encontraron en el juego una forma de motivarla.

Palabras clave: COVID-19; Educación preescolar; Psicomotricidad

\section{ABSTRACT}

The closing of schools due to the COVID-19 pandemic severely affected preschool children, since they were the most vulnerable because they depended on an adult to guide them in their academic activities; therefore, their normal school development was interrupted. The objective of this study is to explain the limitations of psychomotor skills in the preschool stage during COVID-19. Using the descriptive method of bibliographic review, the consultation of the academic literature provided as results coincidences in two topics: psychological and parental difficulties to help them in the development of their psychomotor activities. It was concluded that there were limitations to develop psychomotor skills; however, teachers adapted to the new normality and found in the game a way to motivate them.

Key words: COVID-19; Preschool education; Psychomotor skills

\section{RESUMO}

O fechamento de escolas devido à pandemia da COVID-19 afetou severamente as crianças em idade pré-escolar, pois elas eram as mais vulneráveis, pois dependiam de um adulto para orientá-las em suas atividades acadêmicas, e seu desenvolvimento escolar normal foi interrompido. $\mathrm{O}$ objetivo deste estudo é explicar as limitações das habilidades psicomotoras na fase pré-escolar durante a COVID-19. Usando o método descritivo de revisão bibliográfica, a consulta da literatura acadêmica produziu coincidências em duas áreas: dificuldades psicológicas e parentais em ajudá-los no desenvolvimento de suas atividades psicomotoras. Concluiu-se que havia limitações no desenvolvimento das habilidades psicomotoras; no entanto, os professores se adaptaram à nova normalidade e encontraram em jogo uma forma de motivá-los.

Palavras-chave: COVID-19; Educação pré-escolar; Habilidades psicomotoras 


\section{INTRODUCCIÓN}

El año 2020 debido a la aparición del COVID-19, el gobierno peruano estableció medidas sanitarias para disminuir los contagios, implementando el confinamiento obligatorio o cuarentena; esto significó el cambio del normal desarrollo en las actividades, entre ellas la educación, por lo cual se cerraron las escuelas; trayendo consigo serias dificultades que afectaron estudiantes de colegios, institutos y universidades, en informe presentado por la Defensoría del Pueblo describió que, este contexto afectó el derecho a la educación, develando problemas como fueron las brechas educativas en los sectores pobres, poblaciones rurales y estudiantes en edad preescolar (Defensoría del Pueblo, 2020), además, de acuerdo a cifras de la Organización de las Naciones Unidas para la Educación la Ciencia y la Cultura (UNESCO) publicadas en Coalición por la Educación (2020) esta pandemia provocó en Perú que 8354.077 interrumpieron sus aprendizajes; por tal razón, para no parar el sistema educativo, el Ministerio de Educación implementó la educación remota o a distancia.

En este contexto fue necesario dar una mirada de las complicaciones que tuvo la pandemia, principalmente en los más pequeños, que son los niños de primer y segundo ciclo, cuyas edades oscilan entre los 2 y seis años, al suspenderse las clases presenciales buena cantidad padres, prefirieron cortar el vínculo con sus colegios, desestimando la importancia de su formación inicial, entre los factores que determinaros esta decisión estuvieron las limitaciones de conectividad y uso de las Tecnologías de la Información y la Comunicación (TIC) sin embargo, existió otro grupo que decidieron acompañar en este nuevo escenario educativo a sus hijos, porque comprendieron lo importante de la educación pre escolar en los niños, debido a que ayuda a fortalecer su desarrollo físico y emocional, además, es en esta etapa donde adquiere los primeros conocimientos, habilidades y destrezas (Andrade y Gonzales-Sánchez, 2021).

Durante la educación pre escolar el desarrollo de la psicomotricidad resulta fundamental, cuyas actividades deben ser organizadas sistemáticamente, porque contribuyen al fortalecimiento en lo emocional, físico y psicológico (Arias et al., 2020) el movimiento del cuerpo es importante viéndose en el área educativa, reeducativa y terapéutica la cual actúa en forma integral, se recomendó desde el MINEDU, propiciar ambientes en los hogares para desarrollar las actividades de psicomotricidad, además mediante la trasmisión de los programas "Aprendo en casa" se brindaron pautas sencillas en las cuales las familias podían ayudar a los niños a realizar ciertas actividades; por otro lado, las instituciones de gestión privada con el apoyo de sus docentes utilizaron mucha creatividad incluyendo el uso de las TIC, promoviendo actividades recreativas para poder mejorar la psicomotricidad, los aliados fueron los padres de familia o adultos, orientándolos, consiguiendo los implementos necesarios fáciles de encontrar e casa y motivándolos. Esta integración favoreció al estado de ánimo de los niños frente al contexto complicado que significó la pandemia.

La psicomotricidad tiene sus implicancias en el aspecto emocional, los niños y niñas están repletos de energía, son inagotables, en este periodo de edad fantasean, dejan volar su imaginación, crean sus propias historias mágicas que a sus personajes le dan diferentes roles. Asimismo, se tiene en el 
aspecto motor, debido a que la motricidad gruesa y fina cumplen un rol esencial en los niños y niñas de preescolar, ellos pueden realizar las actividades de psicomotricidad como saltar, escribir manejando el lápiz, dibujar, saltar sobre un pie, encajar, abrochar, tienen mayor coordinación, cortar con la tijera con mucha precisión, comparten juegos con sus compañeros, como juegos solitarios en algunos momentos de su vida, además el "dominio de los movimientos, que tiene lugar en el primer año de vida, resulta de vital importancia para el desarrollo cognoscitivo" (Vol et al., 2019, p. 224). Finalmente, también ayuda en el lenguaje, porque en este periodo los niños y niñas comienzan con algunas interrogantes el "como" y el "por qué" de las diversidades de las cosas de su entorno; inician con los juegos de palabras, combinan hechos, oraciones cortas; hablan mucho acerca de lo que le ocurrió en la escuela, cuando observan imágenes, son capaces de contar historias con su secuencia de los hechos por eso el lenguaje está ligado a la psicomotricidad para poder compartir a través de las sus diversas actividades (Rodríguez et al., 2017).

De acuerdo a lo expuesto, se formuló como objetivo, explicar las limitaciones de la psicomotricidad en la etapa preescolar durante el COVID-19. Esta investigación se consideró importante, porque fue realizada en el contexto de la emergencia sanitaria, que permitirá a futuros investigadores realizar comparaciones, de cómo fue evolucionando el tema con respecto a la situación de los niños en edad pre escolar, además, si se tomaron mediadas o acciones por parte de los gobiernos para reparar las consecuencias dejadas por la pandemia en los niños.

\section{MÉTODO}

El método empleado fue de revisión descriptiva el cual Vera, (2009) manifiesta quien citó a Squires (1994) debido a que este tipo de investigaciones facilitan a los lectores conceptos útiles; para lo cual se consultó 20 artículos científicos que fueron buscados en base de datos: Scopus, Web Of Science (Wos), Education Resources Information Center (Eric) y Scientific Electronic Library Online (Scielo); los dos primeros con acceso restringido, para lo cual se empleó la cuenta institucional que facilita la universidad y los dos últimos de libre acceso.

Se tomó como criterio de inclusión al realizar la búsqueda que las publicaciones hayan sido realizadas entre los años 2020 y 2021 en el contexto de la pandemia, además, que al menos tuvieran una de las palabras clave de interés, las cuales fueron educación preescolar, psicomotricidad, pandemia y COVID-19, además que fueran de acceso abierto publicadas en inglés o español.

El criterio de exclusión fue desestimar publicaciones anteriores a la emergencia sanitaria y que no abordaran el tema desde una perspectiva educativa o psicológica.

\section{DESARROLLO Y DISCUSIÓN}

De acuerdo a la literatura académica consultada, se encontraron coincidencias con respecto a la situación de los niños en edad preescolar durante el confinamiento obligatorio que pudieron ser limitantes para el desarrollo de las actividades psicomotoras; estas fueron dificultades psicológicas 
que causó la inmovilización obligatoria (Sánchez, 2021) generando estrés y temores, les costó comprender los cambios abruptos que se dieron en su familias a causa del encierro, (Massó, 2021) los padres tuvieron complicaciones para hacerles entender de forma sencilla lo que estaba sucediendo en su hogares, además las noticias sobre el fácil contagio del virus incrementaron la angustia.

En la etapa pre escolar es importante que los niños y las niñas puedan socializar para desarrollar en el futuro una adecuada convivencia, asimismo, la comunicación también ayuda a un mejor estado de ánimo y fortalece sus emociones (Etchebehere Arenas et al., 2021) sin embargo, el confinamiento obligatorio afectó la interacción social entre ellos al no poder ir a su escuela, parques o lugares de recreación a los cuales estaban acostumbrados. alterando su normal formación (Duran, 2021) incrementaron el retraso del desarrollo de los niños durante la pandemia (Guijarro, 2021). Otro limitante a parte del encierro fue la exposición de los niños a dispositivos como los celulares, tabletas o computadora, si bien fueron necesario para sus clases a distancia, su uso incrementó el sedentarismo y la dependencia por estos equipos. limitando la actividad física (Rengel y Calle. 2020).

Esta situación preocupó a los padres de familia por las implicancias a futuro con respecto al desarrollo social de sus hijos (Stites et al., 2021), el encierro provocaron dificultades psicológicas, que se manifestaron en su salud mental y física (Abufhele y Jeannere; De Laia Almeida et al., 2021) incrementándose los casos de insomnio y dolores de cabezas, la pandemia exacerbo también condiciones de enfermedades pre existentes afectando en los niños (Palacio-Ortiz et al., 2020), es muy alta la probabilidad de las escuelas psicológica que dejará en ellos la inmovilización obligatoria, si las afecciones mentales no son tratadas a tiempo en los niños podrán tener consecuencias en el futuro (Reséndiz-Aparicio, 2021).

Desde la percepción de los niños este encierro les permitió al principio estar más tiempo con sus padres lo que les causó satisfacción: sin embargo, conforme fueron pasado los días y no poder movilizarse les provocó enojo, irritabilidad, miedo, desgano y preocupaciones (Etchebehere Arenas et al., 2021; Rusca-Jordán et al., 2020).

La segunda coincidencia que se encontró en la literatura fue las dificultades en los padres de poder realizar las actividades académicas y motoras que recomendaban los docentes durante la educación a distancia, independiente de haberse puesto a disposición en distintos países plataformas y programas televisivos (Vallejos y Guevara, 2021) sin embargo, muchos padres de familia sintieron frustración al no poder ayudar sus hijos les generó situaciones de estrés (Martins et al., 2021), ellos necesitaban algunas habilidades digitales para poder conectar a sus hijos en las clases a distancia o remota las cuales muchos desconocían fue un aprendizaje progresivo el irse adaptando conforme los meses del encierro avanzaba y se familiarizaban con la nueva forma de educación (Janampa et al., 2021).

Esta pandemia dejó reflexiones, una de ellas fue pensar en el futuro si otra situación similar se producía, era necesario los países estar preparados para que no afecten nuevamente el sistema educativo (Kim et al., 2021). Para Toran et al., (2021) quienes estudiaron el tema en China y Turquía, realizando entrevistas a padres, 
encontraron que, durante la emergencia sanitaria se dieron aspectos positivos como poder estar más tiempo con sus hijos observando su progreso académico y negativos asumir el rol de docente en el hogar. Es en este contexto que los maestros debieron generar estrategias para ser empleadas en la educación a distancia o remota para fortalecer la psicomotricidad diseñando actividades que encontraron en el juego una alternativa (İnan, 2020), estas tendrían secuencias sencilla para seguir por los padres o personas que acompañaban al niño; asimismo favorecían a la recreación, las actividades lúdicas tienen efectos positivos en los niños mejorando sus emociones (Posso Pacheco et al., 2021), coincidieron con (Rosales et al., 2020) quienes en Chile brindaron recomendaciones para que los niños tuvieran actividad física para mejorar su salud física y mental; en Perú el mes de setiembre del año 2021 se promovió el regreso progresivo y flexible a las escuelas, por lo que se priorizó la actividad motriz y recreativa para mejorar la adaptación de los niños en la nueva normalidad.

\section{CONCLUSIÓN}

Se concluyó que existieron limitaciones de la psicomotricidad la etapa preescolar durante el COVID-19, de acuerdo a literatura académica este encierro los afectó psicológicamente en sus emociones, los padres sintieron frustración $y$ dificultades para poder enseñar a sus hijos en sus hogares, para preservar la continuidad los maestros comprendieron que la mejor forma resultó ser el juego, por tal razón la actividad física puedo ayudar en su salud física y mental. Desde la virtualidad el ingenio de los maestros puede brindar recomendaciones sencillas las cuales los niños puedan realizar como gestos, desplazamientos o juegos.

Finalmente, el progreso de los niños en edad preescolar tiene que adaptarse a la nueva normalidad, por tal razón los padres y docentes deben generar alianzas y espacios de comunicación en favor de los pequeños.

\section{REFERENCIAS}

Abufhele, M., y Jeanneret, V. (2020). Home confinement: The other side of the epidemic. Revista Chilena de Pediatria, 91(3), 319-321. https://doi.org/10.32641/rchped.v91i3.2487

Andrade, J., y Gonzales-Sánchez, A. (2021). Fortaleciendo la conciencia ambiental en estudiantes de Educación Inicial. Propuestas Educativas. https://propuestaseducativas.org/ index.php/propuestas/article/view/705

Arias Huánuco, J. M., Mendivel Gerónimo, R. K., y Uriol Alva, A. del P. (2020). La psicomotricidad en la preescritura de los niños de 5 años de las instituciones educativas de inicial del cercado de Huancavelica. Conrado, 16(76), 43-50

CEPAL-UNESCO. (2020). La educacion en tiempos de la pandemia COVID-19. Comisión Económica Para América Latina y El Caribe, Santiago Oficina Regional de Educación Para América Latina y El Caribe de La Organización de Las Naciones Unidas Para La Educación La Ciencia y La Cultura, 11, 11-13. https:// unesdoc.unesco.org/ark:/48223/pf000037407 5 ? posInSet $=1 \&$ queryId $=9$ ccf $4 a 39-7 c 50-43 e 4-$ 856b-a09632daa7a2

De Laia Almeida, I., Rego, J., Teixeira, A., y Moreira, M. (2021). Social isolation and its impact on child and adolescent development: a systematic review. Revista Paulista de Pdediiatría, 1. https://www.scielo.br/j/rpp/a/ ZjJsQRsTFNYrs7fJKZSqgsv/?lang=en

Defensoría del Pueblo. (2020). Educacion En Pandemia. https://www.defensoria.gob.pe/ wp-content/uploads/2020/08/Serie-Informes- 
Especiales-No-027-2020-DP-La-educaciónfrente-a-la-emergencia-sanitaria.pdf

Duran, M. (2021). The Effects of COVID-19 Pandemic on Preschool Education. International Journal of Educational Methodology, 7(2), 249260. https://doi.org/10.12973/ijem.7.2.249

Etchebehere Arenas, G., León Siri, R. D. De, Silva Paredes, F., Fernández Hernández, D. S., y Quintana Sassone, S. M. (2021). Perceptions and emotions in light of a pandemic: gathering the voices of boys and girls from a public early childhood institution in Uruguay TT - Percepciones y emociones ante la pandemia: recogiendo las voces de niños y niñas de una institución de educa. Psicol. Conoc. Soc, 11(1), 5-23. http:// www.scielo.edu.uy/scielo.php? script $=$ sci_ arttext\&pid=S1688-70262021000100005

Guijarro, E. M. (2021). Childhood and the pandemic: Chronicle of an absence foretold. Salud Colectiva, 17, 1-12. https://doi.org/10.18294/ sc. 2021.3303

İnan, H. Z. (2020). Restructuring Early Childhood Education during the Covid-19 Pandemic. Milli Egitim, 49(1), 831-849. https://doi. org/10.37669/milliegitim.754307

Janampa, E., Gonzales-Sánchez, A., Bayona, G., y Valqui, J. (2021). Acompanhamento familiar aos alunos durante a pandemia COVID-19.pdf. Laplage Em Revista. https://doi.org/https://doi. org/10.24115/S2446-6220202173C1502p.43-50

Kim, J. H., Araya, M., Hailu, B. H., Rose, P. M., y Woldehanna, T. (2021). The Implications of COVID-19 for Early Childhood Education in Ethiopia: Perspectives from Parents and Caregivers. Early Childhood Education Journal, 49(5), 855-867. https://doi.org/10.1007/s10643021-01214-0

Martins, C. R., Neiva, A. C. L., Bahia, A. F., Oliveira, C. X., Cardoso, M. I. S., y Abreu, J. N. S. (2021). Parents' mental health and children's emotional regulation during the COVID-19 pandemic. Psicologia - Teoria e Prática, 23(1), 1-19. https:// doi.org/10.5935/1980-6906/eptpc1913534
Massó, E. (2021). Infancia y pandemia: Crónica de una ausencia anunciada. Salud Colectiva, 17, 3303. https://doi.org/10.18294/sc.2021.3303

Palacio-Ortiz, J. D., Londoño-Herrera, J. P., Nanclares-Márquez, A., Robledo-Rengifo, P., y Quintero-Cadavid, C. P. (2020). Trastornos psiquiátricos en los niños y adolescentes en tiempo de la pandemia por COVID-19. Revista Colombiana de Psiquiatría, 49(4), 279-288. https://doi.org/10.1016/j.rcp.2020.05.006

Posso Pacheco, R. J., Otáñez Enríquez, N. R., Cóndor Chicaiza, J. D. R., Cóndor Chicaiza, M. G., y Lara Chala, L. D. R. (2021). Educación Física remota: juegos motrices e inteligencia kinestésica durante la pandemia COVID-19 TT - Educação física à distância: jogos motores e inteligência quinestésica durante a pandemia de COVID-19 TT - Remote Physical Education: motor games and k. Podium. Revista de Ciencia y Tecnología En La Cultura Física, 16(2), 564-575. http://scielo.sld.cu/ scielo.php?script=sci_arttext \&pid $=$ S199624522021000200564\&lang $=\mathrm{pt}$

Rengel, M. y Calle, I. (2020) Impacto psicológico de la pandemia del COVID 19 en niños. Revista de Psicología Nro. Especial http:// www.scielo.org.bo/scielo.php? script $=\mathrm{sci}_{-}$ arttext\&pid=S2223-3032202000030001

Reséndiz-Aparicio, J. C. (2021). How the COVID-19 contingency affects children. Boletin Medico Del Hospital Infantil de Mexico, 78(3), 216-224. https://doi.org/10.24875/BMHIM.20000140

Rodríguez, M., Gómez, I., Prieto Ayuso, A., y Pedro, G. (2017). La educación psicomotriz en su contribución al desarrollo del lenguaje en niños que presentan necesidades específicas de apoyo educativo. Revista de Investigación En Logopedia.

Rosales, C. K., Erazo, P. V., Valderrama, J. F., González, J. B., Terneus, D. H., Stagno, R. U., Sarquis, F. J., Reyes, A. S., Miranda, F. V., Plaza, R. S., y Contreras, L. V. (2020). Sport COVID-19 orientations: Recommendations for return to physical activity and sports in children and adolescents. Revista Chilena de Pediatria, 91(7), 1-16. https://doi.org/10.32641/rchped. 
vi91i7.2782

Rusca-Jordán, F., Cortez-Vergara, C., TiradoHurtado, B. C., y Strobbe-Barbat, M. (2020). Una aproximación a la salud mental de los niños, adolescentes y cuidadores en el contexto de la COVID-19 en el Perú. Acta Medica Peruana, 37(4), 556-558. https://doi.org/10.35663/ amp.2020.374.1851

Sánchez Boris, I. M. (2021). Impacto psicológico de la COVID-19 en niños y adolescentes. Medisan, 25(1), 123-141.

Stites, M. L., Sonneschein, S., y Galczyk, S. H. (2021). Preschool Parents' Views of Distance Learning during COVID-19. Early Education and Development, 32(7), 923-939. https://doi.or $\mathrm{g} / 10.1080 / 10409289.2021 .1930936$

Toran, M., Sak, R., Xu, Y., Şahin-Sak, İ. T., \& Yu, Y. (2021). Parents and children during the COVID-19 quarantine process: Experiences from Turkey and China. Journal of Early Childhood Research, 19(1), 21-39. https://doi. org/10.1177/1476718X20977583

Vallejos, G., y Guevara, C. (2021). Educación en tiempos de pandemia. Revista Conrado. https:// emea.mitsubishielectric.com/ar/productssolutions/factory-automation/index.html

Vera, O. (2009). Artículos de Revisión. Revista Médica, 58(02), 033-033. http:// www.scielo.org.bo/scielo.php? script $=$ sci arttext\&pid=S1726-89582009000100010

Vol, M., Vald, C., Vald, C. C., Dupeyr, N., y Sa, H. (2019). The development and of fine motor skills in pre-school children. Mendive. Revista de Educación, 17(2), 222-239 